\title{
Exploración psicométrica sobre deficiencia motivacional en consumidores de marihuana
}

\section{Psychometric exploration of motivational deficit in marijuana users}

\author{
Orlando Scoppetta DG ${ }^{1}$, Guillermo A. Castaño Pérez ${ }^{2}$, Elisardo Becoña Iglesias ${ }^{3}$
}

\author{
y Sandra Milena Restrepo Escobar ${ }^{4}$
}

${ }^{1}$ Psicólogo. Especialista en Análisis de Datos. Especialista en Docencia Universitaria. Magister en Estudios de Población. Estudiante de Doctorado en Psicología, Universidad Católica de Colombia, Bogotá. E-mail: oscoppetta18@ucatolica.edu.co, orlando.scoppetta@gmail.com

${ }^{2}$ Médico. Especialista en Farmacodependencia. Especialista en Patología Dual. Magister en Drogodependencias. PhD. en Psicología de la Salud. Coordinador de Maestría en Drogodependencias y docente en la Facultad de Medicina, Universidad CES, Medellín, Colombia.

\section{E-mail: gacastano@ces.edu.co}

${ }^{3}$ Licenciado en Filosofía y Ciencias de Educación (Sección de Psicología). PhD. en Psicología. Catedrático de Psicología Clínica en la Facultad de Psicología, Departamento de Psicología Clínica y Psicobiología, Universidad Santiago de Compostela, España.

E-mail: elisardo.becona@usc.es

xPsicóloga. Especialista en Farmacodependencia. Magister en Educación y Desarrollo. Docente de la Facultad de Psicología y Ciencias Sociales, Universidad Católica Luis Amigó, Medellín,

Colombia. E-mail: sandra.restrepoes@amigo.edu.co

\begin{abstract}
Los subsidios económicos para esta investigación fueron otorgados por la Universidad CES de Medellín, la Universidad Santiago de Compostela y la Universidad Católica Luis Amigó. Los autores agradecen a la Asociación Cannábica de Colombia por facilitar la convocatoria de los participantes en este estudio y a la doctora Alba Lucía Meneses sus recomendaciones para el análisis de los datos.

Universidad CES, Medellín, Colombia.
\end{abstract}

\section{Resumen}

Si bien es común ver en la práctica clínica deficiencias en la motivación en usuarios de marihuana, no ha sido firmemente establecida la existencia de un posible síndrome amotivacional asociado con el consumo de esta droga, entre otras razones por la concurrencia de factores como la depresión que pueden llevar a generalizar inadecuadamente la situación de algunos usuarios de la sustancia. Esta investigación se orientó a obtener evidencia acerca de la existencia de deficiencias en la motivación en consumidores de marihuana. Se construyó una batería de preguntas a partir de una revisión y una consulta a expertos, que fue aplicada a una muestra de 1455 participantes, junto con otros instrumentos seleccionados para excluir del análisis a los participantes con depresión, síntomas prefrontales, trastornos por uso de alcohol, apatía y aplanamiento afectivo, que pudieran confundir 
los resultados. Se aplicaron métodos estadísticos y psicométricos basados en el modelo de Rasch, para establecer qué comportamientos serían más característicos de una deficiencia motivacional en consumidores habituales de marihuana. Se estimó que un $54.9 \%$ de los participantes tendría un trastorno grave por uso de marihuana, un $54.0 \%$, síntomas de aplanamiento afectivo y un $60.9 \%$ serían usuarios de marihuana con altos niveles de tetrahidrocannabinol (THC). La media de cigarrillos de marihuana consumidos al mes sería de 44.5. Mediante el modelo de Rasch se estableció que con el conjunto de preguntas construido era posible identificar una dimensión o factor coincidente con una deficiencia motivacional. Se encontró evidencia que sugiere la existencia de apatía emocional; retraimiento social; descuido de la imagen personal y enlentecimiento motor.

Palabras clave: motivación; marihuana; drogas ilícitas; consumo de drogas; Modelo de Rasch; psicometría; análisis factorial.

\section{Abstract}

It is common to find marijuana users with alterations in motivation, in clinical practice. Several studies have found evidence about a possible amotivational syndrome in marijuana users, both from the analysis of behavior and from the neurological examination. However, the existence of such a syndrome associated with the use of marijuana has not been firmly established, among other reasons, due to the concurrence of factors such as depression and the use of other drugs that may lead to inadequate generalization of the situation of some consumers of the substance and due to methodological difficulties in research aimed at establishing whether or not there is a decrease in the general motivation associated with the use of marijuana. Likewise, the decrease in motivation could be the result of the disorder by substances rather than by the consumption of marijuana. Due to the growing use of marijuana in the world, the study of its consequences is of the greatest importance, for the design of better treatments for people with substance use disorders. The aim of this study was to obtain evidence about the existence or not of deficiencies in the motivation of marijuana users. In addition, we sought to establish which are the characteristic behaviors that show this possible reduction of motivation. A battery of questions was constructed from a systematic literature review in PubMed, Lilacs, MedLine, Cochrane library, Embase, Science Direct and Bibliomed, using as terms "amotivational syndrome", "amotivation cannabis", "amotivation marijuana", "comorbidity cannabis", "comorbidity marijuaba", "mental disorders and cannabis", "cannabis and mental consequences", "amotivation and drug use". Likewise, an expert consultation was done about behaviors that were indicative of a reduction in the motivation of marijuana users. From the review and consultation of experts, a set of 70 possible behaviors expressed in questions about probable deficiencies in motivation was extracted. These questions, along with other instruments selected to exclude from the analysis participants with depression, prefrontal symptoms, alcohol use disorders, apathy and affective flattening, which could confuse the results, were presented to a sample of 1455 participants. The Rasch model was applied to establish whether it was possible to identify a measurable and consistent dimension that corresponded to a deficiency in motivation, and to discern which behaviors would be most characteristic of a possible motivational deficiency in habitual marijuana users. In addition, an exploratory factor analysis was carried out to identify possible groupings of the characteristic symptoms of a reduction in motivation. Our Results and conclusions show that it was estimated that $54.9 \%$ of the participants would have a serious disorder due to the use of marijuana; $54.0 \%$ presented symptoms of affective flattening, and $60.9 \%$ would be marijuana users with high levels of tetrahydrocannabinol (THC). The average number of 
cigarettes consumed per month would be 44.5 . Using the Rasch model it was established that, with the constructed set of questions, it was possible to identify a dimension or factor coinciding with a motivational deficiency. Using the exploratory factor analysis, it was found that the characteristic behaviors could be grouped into four possible subdimensions: emotional apathy; social withdrawal; neglect of personal image and motor slowdown.

Keywords: motivation; drug abuse; street drugs; Rasch Model; psychometry; factor analysis; marijuana.

\section{Introducción}

McGlothlin y West (1968) y Smith (1968) describieron que entre los consumidores de marihuana se presentaba un conjunto de síntomas caracterizados por falta de motivación y energía. Los primeros, a partir de estudios de casos con universitarios y el segundo, con base en una investigación con estudiantes de secundaria.

A partir de allí, diversos autores han puesto de relieve la existencia de un síndrome amotivacional entre consumidores de marihuana. Los síntomas relatados tienen que ver con la pasividad y la indiferencia persistentes, la incapacidad para desarrollar planes futuros, el empobrecimiento afectivo, el abandono del cuidado personal, la inhibición sexual, la anergia y la pereza (Bobes y Calafat, 2000; Gold, 1991; Gutiérrez-Rojas, De Irala y Martínez-González, 2006; Hall, Solowij y Lemon, 1994; Tziraki, 2012), el deterioro de las relaciones sociales, la pérdida del impulso y la motivación, el desapego emocional, el alejamiento de la realidad y la reducción en la atención y la memoria (Rovai et al., 2013).

Sin embargo, aunque es frecuente el reporte por parte de clínicos de una acusada falla en la motivación por parte de usuarios de marihuana, expresada por una actitud indiferente frente a la vida y aplazamiento de tareas, no hay certidumbre acerca de la existencia de un síndrome amotivacional entre consumi- dores de cannabis, entre otras razones porque los signos que indican el probable síndrome pueden ser el resultado de la intoxicación aguda frecuente por parte de consumidores habituales (Karila et al., 2014), o por otros factores que tengan en común la afectación de la motivación, como la depresión, el alcoholismo o el consumo de otras drogas. En este sentido, Lawn et al. (2016) encontraron que el uso de marihuana produce un efecto amotivacional pasajero, pero no pudieron sustentar la hipótesis de un efecto negativo y persistente sobre la motivación.

Otros autores niegan de plano la existencia del síndrome en cuestión (Barnwell, Earleywine y Wilcox, 2006), indicando que se trata de una generalización inadecuada de observaciones clínicas o de análisis no profundos de datos, aunque existe evidencia neurofisiológica de que el consumo continuado de marihuana afecta la motivación, vía alteración del sistema de recompensas (van Hell et al., 2011; Volkow et al., 2014; Volkow et al., 2016)

Dentro de los problemas metodológicos observados en trabajos que han hecho referencia al síndrome amotivacional, Barnwell, Earleywine y Wilcox (2006), sin negar su existencia, han reportado que muchos de los trabajos han incluido pequeñas muestras, no han sido muestras puras de solo consumidores de cannabis, no han descartado comorbilidad que pudiera producir amotivación (depresión, apatía, trastornos mentales, disfunción ejecutiva), no han sido consistentes con el tipo de consumidores en cuanto a la frecuencia y la cantidad de consumo (consumo pesado o liviano), no han tenido en cuenta la edad de inicio en el consumo, ni el tipo de marihuana consumida (niveles de $\Delta$ 9-tetrahidrocannabinol).

Hasta el momento, no existe consenso acerca de la existencia de un síndrome específico en consumidores de marihuana. Esto es porque se asume que la exposición continuada a las drogas, no solamente a la marihuana, produce alteraciones del circuito cerebral de 
recompensas, lo que a su vez tiene efectos sobre la motivación (Calipari et al., 2017). Así que, aunque se aceptara la existencia del síndrome amotivacional, no se consideraría apropiado afirmar que se trata de una condición particular inducida por el uso de marihuana (Rovai et al., 2013; Solé Puig, 2001).

En la dilucidación de la etiología del síndrome amotivacional, Lac y Luk (2017) encontraron que el consumo de marihuana producía una disminución en la iniciativa y la persistencia, no así el alcohol. Adicionalmente, en la búsqueda de las bases de un síndrome específico, van Hell et al. (2011), en un estudio con resonancia magnética funcional, encontraron que el uso de cannabis producía una reducción de la actividad de activación de la recompensa en el núcleo accumbens, en comparación con el consumo de nicotina, lo que sugiere una afectación diferencial por esta sustancia. Desde la perspectiva de Volkow et al. (2016), si bien está establecido que hay efectos negativos del cannabis sobre la motivación, no está claro si esto es consecuencia del uso del cannabis en sí mismo o del trastorno por uso de sustancias.

Aunque el consumo de marihuana es el principal factor que determina el crecimiento del uso de drogas en Colombia (Gobierno de Colombia, 2018; Scoppetta, 2010), hasta el momento no se tiene conocimiento acerca de estudios que profundicen en las implicaciones de la motivación del consumo de esta sustancia.

Con el propósito de identificar factores característicos de deficiencia motivacional en consumidores de marihuana que pudieran ser útiles en la detección del síndrome amotivacional, se aplicó un cuestionario construido a partir de una revisión sistemática de literatura y de una consulta a expertos clínicos.

\section{Método}

\section{Participantes}

La muestra estuvo constituida por 1455 participantes de la Asociación Cannábica de Colombia, todos consumidores de marihuana. E1 $78.3 \%$ eran hombres y el $21.7 \%$, mujeres (1139 y 316, respectivamente). Sus edades estaban entre 18 y 35 años, con una media general de 22.7 años (igual para hombres y mujeres) y una desviación estándar de 4.1.

La gran mayoría de los participantes (83.4 $\%$ ) informaron que eran personas solteras; un $33.7 \%$ dijo ser únicamente estudiante y un $32.3 \%$, dedicarse simultáneamente al estudio y al trabajo. Un $48.2 \%$ dijo tener estudios universitarios, en curso o ya terminados. Casi la mitad de los participantes se ubicó a sí mismo en el estrato socioeconómico 3 , y el $27.2 \%$, en el estrato 2 .

\section{Instrumentos}

Para la identificación de síntomas característicos de deficiencia en la motivación, se construyó un cuestionario a partir de una revisión sistemática de literatura, que permitió la identificación de posibles indicadores de deficiencia motivacional en las siguientes bases de datos PubMed, Lilacs, MedLine, Cochrane library, Embase, Science Direct y Bibliomed, utilizando los siguientes términos descriptores: "amotivational síndrome", "amotivation cannabis", "chronic cannabis consumption", "comorbidity cannabis", "mental disordes and cannabis", "cannabis and mental consequences", "amotivation and drug use" y similares en idioma español. La búsqueda se realizó entre los meses de febrero y mayo de 2016. Lo encontrado en la literatura se redactó a manera de ítems, y se construyó un cuestionario de 70 reactivos, que luego fue sometido a la revisión de cinco expertos clínicos, tres psiquiatras y dos psicólogos con estudios en drogodependencias y experiencia clínica en la atención de pacientes consumidores de drogas 
por más de 5 años. De esta revisión surgieron 56 ítems que conformaron el conjunto de reactivos relacionados con fallas en la motivación. Se excluyeron aquellos ítems que al menos no fueran consensuados por tres de los expertos. Construido el cuestionario final, fue adecuado para su calificación e interpretación a una escala de frecuencia: nunca, pocas veces, a veces sí a veces no y siempre, asimilables a valores entre 0 y 4 .

Para establecer el grado de dependencia a la marihuana, se utilizaron los criterios del DSM-5 (American Psychiatric Association, 2013), con el propósito de clasificar a los participantes con trastorno por uso de marihuana.

También se usó el inventario de Beck (Beck, Steer, Brown, Sanz y Vázquez, 2011) para identificar participantes con síntomas de depresión. Este inventario ha sido ampliamente estudiado y se han encontrado medidas de consistencia de hasta .92 en poblaciones latinoamericanas (Valdés et al., 2017). Se administró el cuestionario sobre síntomas prefrontales para dar cuenta de los sujetos con esta sintomatología, que en su creación obtuvo indicadores de confiabilidad entre .81 y .86 (Ruiz-Sánchez de León et al., 2012), la Escala de Apatía (Marin, 1991), cuyo autor reportó una confiabilidad test-retest entre .76 y .94 (Marin, Biedrzycki y Firinciogullari, 1991) para identificar apatía y aplanamiento afectivo presente en trastornos mentales, y el cuestionario AUDIT (Alcohol Use Disorders Identification Test; Babor, Higgins-Biddle, Saunders y Monteiro, 2001) para evidenciar trastornos por uso de alcohol. El AUDIT ha sido utilizado ampliamente en diferentes contextos en los cuales sus medidas de confiabilidad han variado entre .80 y .90 (García Carretero, Novalbos Ruiz, Martínez Delgado y O'Ferrall González, 2016; Medina et al., 2014).

\section{Procedimiento}

Los participantes fueron voluntarios seleccionados de la Asociación Cannábica de
Colombia. Se descartó la participación de los sujetos menores de 18 años y mayores de 35 , como de quienes manifestaron haber usado marihuana en la última hora previa a la aplicación de los cuestionarios, o fueran consumidores habituales de otras drogas diferentes a la marihuana, excepto el tabaco. Los instrumentos fueron autoadministrados. Todos los sujetos firmaron un formato de consentimiento informado para participar en el estudio.

\section{Análisis de datos}

Se compararon las medias de los puntajes obtenidos en las respuestas a las preguntas formuladas a los participantes, calculando sus intervalos de confianza de $95 \%$, con el propósito de identificar qué variables se asociaban con diferencias en los puntajes medios. Posteriormente, se hizo análisis con el modelo de Rasch para depurar las preguntas mediante estudio de funcionamiento diferencial y capacidad de discriminación, con la intención de establecer qué conjunto de comportamientos es de mayor utilidad para identificar la posible deficiencia en la motivación de los consumidores consuetudinarios de marihuana, definidos estos como consumidores de al menos medio cigarrillo diario. Finalmente, con el conjunto de ítems restantes se hizo un análisis factorial, con extracción por el método de máxima verosimilitud y rotación Promax con $k=4$ para explorar si estos comportamientos se agrupaban en factores discernibles empírica y conceptualmente.

\section{Resultados}

Con respecto a variables relacionadas con el estado de salud, $95 \%$ de los participantes contestaron que consideraban que su salud era entre excelente y buena. Según la clasificación del DSM-5 para trastorno por consumo de sustancias, un $54.9 \%$ tendría un trastorno grave, $18.1 \%$ moderado, $17.3 \%$ leve y $9.6 \%$ no tendría un trastorno de este tipo. Además, $54 \%$ de los participantes tendría signos 
de aplanamiento afectivo según la escala dispuesta para detectar esta característica.

En cuanto al consumo problemático de alcohol, con el AUDIT se categorizó a los sujetos así: el $54.7 \%$ no tendría problemas con el alcohol y el $45.3 \%$ tendría riesgo de abuso o dependencia. Por su parte, $85.3 \%$ de los participantes tendría un grado de depresión mínima, según el inventario de Beck, un 9.1 $\%$, depresión leve, un $4.3 \%$, depresión moderada y el restante $1.4 \%$, depresión grave.

Entre los participantes en el estudio, el 60.9 $\%$ informó que consumía marihuana del tipo cripa, es decir, marihuana modificada con altos niveles de THC; el 31.8\% consumía marihuana regular y el $7.3 \%$ de otro tipo, en su mayoría producto de auto-cultivos y que también podrían contener niveles elevados de THC. La media de cigarrillos de marihuana consumidos al mes fue de 44.5, y de los días de consumo en el último mes, de 22 . Por su parte, $83.8 \%$ de los participantes dijo consumir marihuana todos los días. Un $40.1 \%$ dijo que consumía un cigarrillo al día, un $22.8 \%$, dos cigarrillos o más, un $21 \%$, uno a dos cigarrillos y $16.1 \%$, menos de un cigarrillo diario.

Como paso seguido, se calculó la sumatoria de los puntajes de las 56 variables sobre motivación. El total de los participantes obtuvo un promedio de 65.8 , de un total posible de 224 puntos, es decir que se ubicaron alrededor de un $29.4 \%$ del total posible. Estos puntajes oscilaron entre 4 y 110 (50\% del total posible). La Tabla 1 muestra el comportamiento del puntaje obtenido en el cuestionario de 56 ítems.

Tabla 1.

Promedios e intervalos de confianza al $95 \%$ en el puntaje de ítems sobre motivación, según variables de interés.

\begin{tabular}{|c|c|c|c|c|c|c|}
\hline \multirow[t]{2}{*}{ Variables } & \multirow[t]{2}{*}{$n$} & \multirow[t]{2}{*}{$M$} & \multirow[t]{2}{*}{ DS } & \multicolumn{2}{|c|}{ Límites de intervalo (95 \%) } & \multirow[t]{2}{*}{ Diferencias } \\
\hline & & & & Inferior & Superior & \\
\hline \multicolumn{7}{|l|}{ Sexo } \\
\hline Hombre & 1139 & 66.2 & 24.1 & 64.8 & 67.6 & \\
\hline Mujer & 316 & 64.6 & 23.1 & 62 & 67.2 & \\
\hline \multicolumn{7}{|l|}{ Edad } \\
\hline 18 a 21 & 725 & 68.4 & 23 & 66.7 & 70.1 & $*$ \\
\hline 22 a 35 & 730 & 63.3 & 24.5 & 61.5 & 65.1 & \\
\hline \multicolumn{7}{|l|}{$\begin{array}{c}\text { Estrato } \\
\text { socioeconómico }\end{array}$} \\
\hline 1 у 2 & 472 & 67.2 & 23.3 & 65.1 & 69.4 & \\
\hline 3 & 682 & 64.6 & 23.8 & 62.8 & 66.4 & \\
\hline 4,5 y 6 & 301 & 66.4 & 25 & 63.6 & 69.2 & \\
\hline \multicolumn{7}{|l|}{ Nivel educativo } \\
\hline Hasta secundaria & 340 & 67.3 & 24.2 & 64.7 & 69.9 & \\
\hline Técnico & 338 & 63.7 & 24.1 & 61.1 & 66.3 & \\
\hline Universitario & 777 & 66.1 & 23.6 & 64.4 & 67.8 & \\
\hline \multicolumn{7}{|l|}{$\begin{array}{c}\text { Tipo de } \\
\text { marihuana }\end{array}$} \\
\hline Regular & 454 & 65.3 & 23.9 & 63.1 & 67.6 & \\
\hline Cripa & 899 & 66.4 & 24.2 & 64.8 & 68 & \\
\hline Otra & 102 & 62.9 & 20.7 & 58.9 & 67 & \\
\hline
\end{tabular}




\begin{tabular}{|c|c|c|c|c|c|c|}
\hline \multirow[t]{2}{*}{ Variables } & \multirow[t]{2}{*}{$n$} & \multirow[t]{2}{*}{$M$} & \multirow[t]{2}{*}{ DS } & \multicolumn{2}{|c|}{ Límites de intervalo (95\%) } & \multirow[t]{2}{*}{ Diferencias } \\
\hline & & & & Inferior & Superior & \\
\hline \multicolumn{7}{|l|}{$\begin{array}{c}\text { Días de } \\
\text { consumo en los } \\
\text { últimos } 30\end{array}$} \\
\hline 0 a 15 & 397 & 65.4 & 23.4 & 62.8 & 67.9 & \\
\hline 16 a 27 & 321 & 67 & 22.1 & 64.8 & 69.2 & \\
\hline 28 a 29 & 117 & 70.8 & 21.5 & 66.9 & 74.8 & \\
\hline 30 & 610 & 64.3 & 25.4 & 62.3 & 66.4 & \\
\hline \multicolumn{7}{|l|}{$\begin{array}{l}\text { Cigarrillos al } \\
\text { día }\end{array}$} \\
\hline Menos de 1 & 234 & 66.7 & 22.7 & 63.8 & 69.7 & \\
\hline 1 & 584 & 64.6 & 23.1 & 62.7 & 66.5 & \\
\hline 1 a 2 & 305 & 65.4 & 24 & 62.8 & 68.2 & \\
\hline 2 y más & 332 & 67.7 & 25.8 & 64.9 & 70.5 & \\
\hline \multicolumn{7}{|c|}{$\begin{array}{c}\text { Cantidad de cigarrillos } \\
\text { en los } 30 \text { dias }\end{array}$} \\
\hline Hasta 12 & 367 & 65.9 & 23.2 & 63.5 & 68.3 & \\
\hline De 12.5 a 29 & 308 & 66.6 & 22.1 & 64.1 & 69.1 & \\
\hline De 30 a 60 & 476 & 64 & 24 & 61.8 & 66.1 & \\
\hline De 65 a 120 & 304 & 67.8 & 26 & 64.9 & 70.8 & \\
\hline \multicolumn{7}{|l|}{$D S M-5$} \\
\hline $\begin{array}{l}\text { Sin trastorno } \\
\text { por consumo de } \\
\text { marihuana }\end{array}$ & 392 & 56.3 & 22 & 54.1 & 58.5 & $*$ \\
\hline $\begin{array}{c}\text { Con trastorno } \\
\text { por consumo de } \\
\text { marihuana } \\
\end{array}$ & 1063 & 69.3 & 23.6 & 67.9 & 70.8 & \\
\hline \multicolumn{7}{|l|}{$A U D I T$} \\
\hline Sin riesgo & 796 & 63.1 & 23.5 & 61.5 & 64.7 & $*$ \\
\hline Riesgo abuso & 417 & 66.6 & 24.2 & 64.2 & 69 & \\
\hline $\begin{array}{c}\text { Riesgo de } \\
\text { dependencia }\end{array}$ & 242 & 73.4 & 22.9 & 70.5 & 76.3 & \\
\hline \multicolumn{7}{|l|}{$\begin{array}{l}\text { Sindrome } \\
\text { prefrontal }^{\text {a }}\end{array}$} \\
\hline $\begin{array}{l}\text { Sin síndrome } \\
\text { prefrontal }\end{array}$ & 1041 & 57.9 & 20.1 & 56.6 & 59.1 & $*$ \\
\hline $\begin{array}{l}\text { Con síndrome } \\
\text { prefrontal }\end{array}$ & 414 & 85.9 & 20.8 & 83.8 & 87.9 & \\
\hline \multicolumn{7}{|l|}{$\begin{array}{l}\text { Aplanamiento } \\
\text { afectivo }^{\mathrm{b}}\end{array}$} \\
\hline $\begin{array}{c}\text { Sin } \\
\text { aplanamiento }\end{array}$ & 669 & 63.1 & 22.4 & 61.4 & 64.8 & $*$ \\
\hline $\begin{array}{c}\text { Con } \\
\text { aplanamiento }\end{array}$ & 786 & 68.2 & 24.8 & 66.4 & 69.9 & \\
\hline
\end{tabular}




\begin{tabular}{|c|c|c|c|c|c|c|}
\hline \multirow[t]{2}{*}{ Variables } & \multirow[t]{2}{*}{$n$} & \multirow[t]{2}{*}{$M$} & \multirow[t]{2}{*}{ DS } & \multicolumn{2}{|c|}{ Límites de intervalo (95\%) } & \multirow[t]{2}{*}{ Diferencia } \\
\hline & & & & Inferior & Superior & \\
\hline \multicolumn{7}{|l|}{ Depresión $^{\mathrm{c}}$} \\
\hline Grave & 20 & 110.6 & 33.6 & 94.8 & 126.3 & $*$ \\
\hline Moderada & 62 & 95.3 & 22.2 & 89.7 & 101 & \\
\hline Leve & 132 & 84.3 & 20.2 & 80.8 & 87.8 & \\
\hline Mínima & 1241 & 61.7 & 21.3 & 60.5 & 62.9 & \\
\hline
\end{tabular}

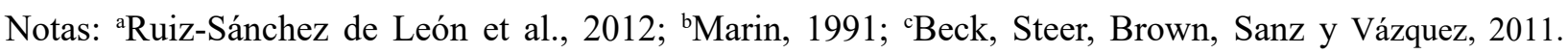
* Diferencia significativa en el intervalo a $95 \%$.

Con el propósito de limitar el efecto de otros eventos en salud sobre la deficiencia motivacional, se excluyeron del análisis subsiguiente los sujetos con depresión, con riesgo de abuso o dependencia al alcohol, aplanamiento afectivo y con síndrome prefrontal. Así, el número de registros efectivos se redujo a 617 .

El siguiente paso consistió en el ajuste de las categorías de respuesta, atendiendo a la recomendación de Bond y Fox (2015), para lograr una separación más clara de las respuestas. Así, las categorías de respuesta nunca y siempre se mantuvieron. Como se muestra en la figura 1, las categorías a veces sí a veces no, pocas veces y muchas veces se colapsaron en una única categoría, logrando una distancia superior a 1.4 entre categorías de respuesta.

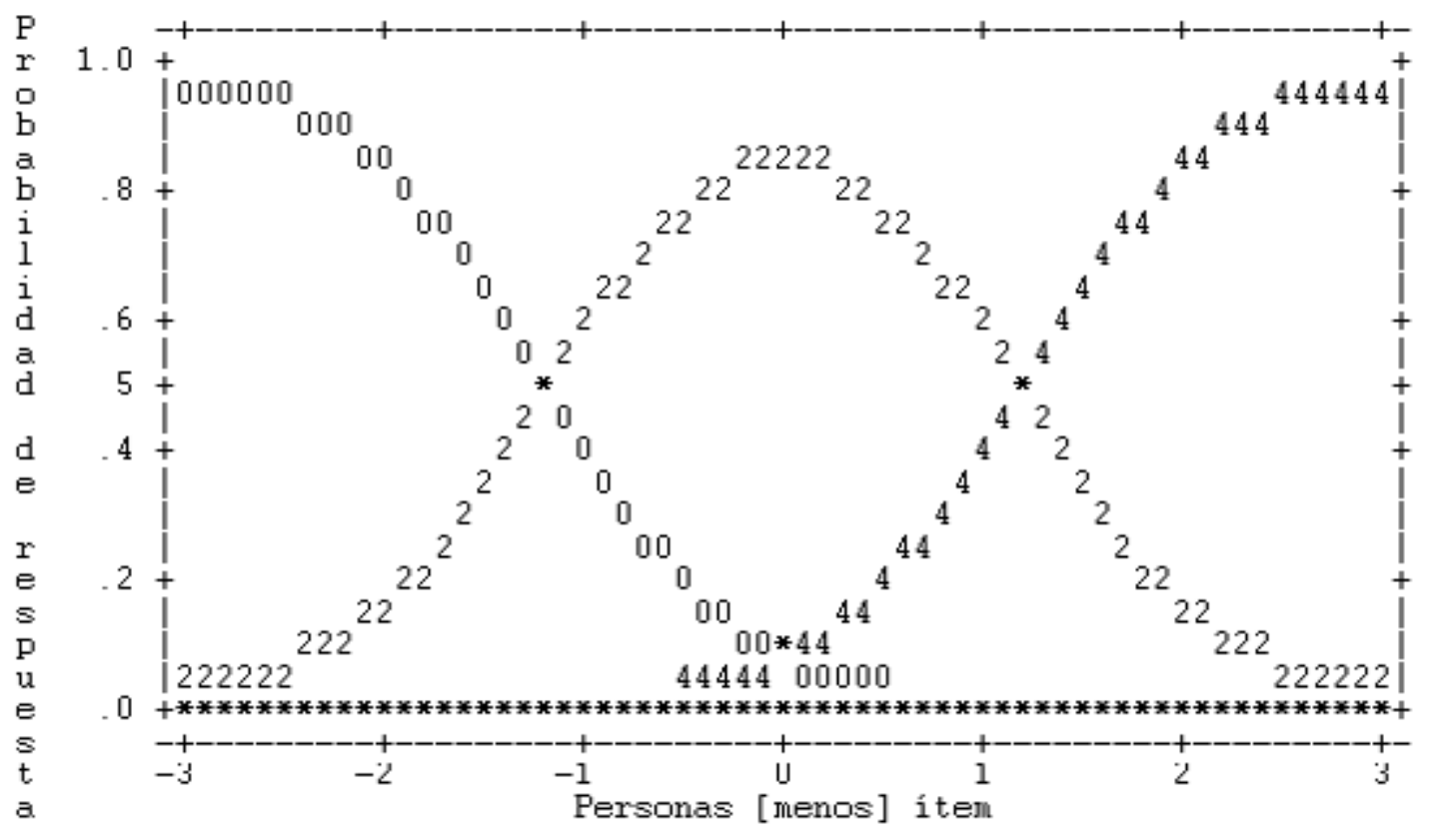

Figura 1. Curvas de probabilidad de las categorías de respuesta colapsadas del cuestionario sobre deficiencia en motivación.

Para identificar si había diferencias en los comportamientos según el sexo, se analizaron los estimadores de Rasch-Welch y el tamaño de la diferencia en las respuestas según el sexo del respondiente. Este análisis mostró que las preguntas 34 ("He recibido críticas de personas cercanas o familiares, porque he abandonado un poco mi aspecto personal"), 
37 ("Estoy menos interesado ahora por el sexo que antes), 39 (Considero el sexo como una necesidad vital para mi vida"), 40 ("Mantengo un alto deseo sexual") y 54 ("Expreso fácilmente mis emociones") mostraron comporta- miento diferencial según el sexo del respondiente (masculino o femenino), por lo que fueron eliminadas. La figura 2 presenta los valores $t$ del análisis de funcionamiento diferencial de los ítems.

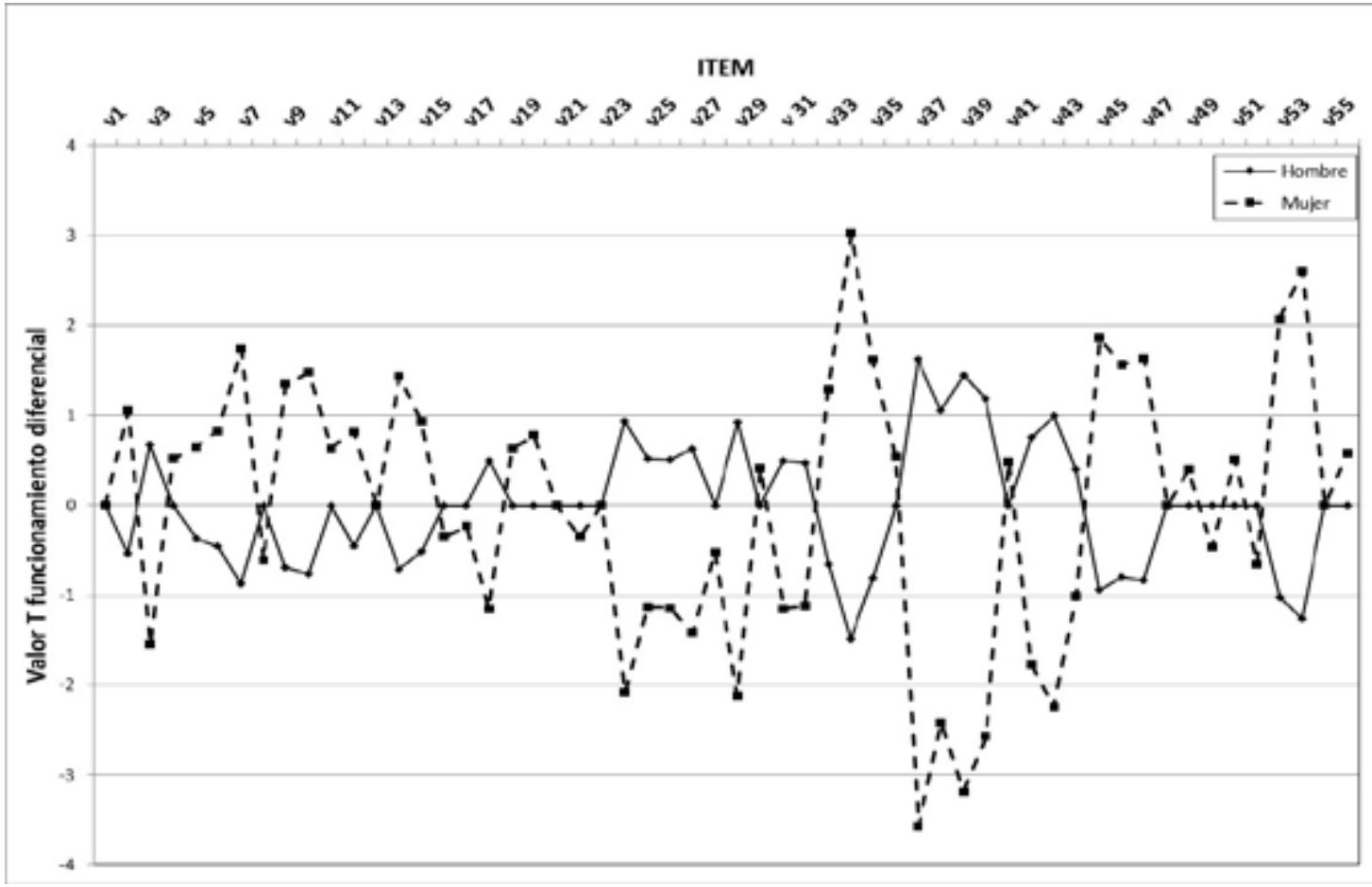

Figura 2. Valor $t$ de la diferencia en el funcionamiento de los ítems del cuestionario sobre deficiencia en motivación.

La pregunta 50 ("Mi círculo de amistades ahora es más cerrado") tuvo un índice de discriminación de solamente .104. Los demás ítems mostraron indicadores de ajuste de acuerdo al modelo. Además, se identificaron y eliminaron 53 participantes por tener puntuaciones por fuera de los rangos del modelo.

Para establecer la existencia de una dimensión o característica latente que pudiera asimilarse a una deficiencia en la motivación, se siguió el criterio planteado por Linacre (2017) expresado así: que la varianza explicada por los ítems sea al menos cuatro veces la varianza del primer contraste; que la varianza explicada de las medidas sea superior al $5 \%$; que el autovalor del primer contraste esté entre tres y 1.5 y que la varianza explicada por el primer contraste sea menor al
$5 \%$. Con las preguntas restantes, la varianza explicada por las medidas fue del $26.3 \%$, la varianza explicada por los ítems fue del $21 \%$ y la varianza del primer contraste del $4.8 \%$ y el autovalor del primer contraste fue de 3.0. Por esto, puede afirmarse que el conjunto de preguntas formuladas a los usuarios de marihuana muestra la existencia de una dimensión latente.

Con la intención de aproximarse a verificar si el conjunto de comportamientos tendría cierto grado de consistencia, se estimó la confiabilidad de las personas en .87 y la de los ítems en .99. Adicionalmente, se encontró un alpha de Cronbach de .89. El índice de separación fue de 2.64.

Finalmente, para discernir de entre las preguntas restantes si existen factores con 
sentido teórico y empírico, se aplicó un análisis factorial. Se estableció que entre cuatro y cinco factores serían la mejor opción en términos de recoger la varianza del sistema; esto confirmado mediante el método de análisis paralelo (DeVellis, 2017).

En la Tabla 2 se presenta la matriz facto- rial. La mejor solución se logró con cuatro factores, mediante extracción con el método de máxima verosimilitud y rotación Promax con $k=4$, de acuerdo con las indicaciones de Morales Vallejo (2013) y Lloret-Segura, Ferreres-Traver, Hernández-Baeza y TomásMarco (2014).

Tabla 2.

Agrupación factorial de items después de la selección con el modelo de Rasch.

\begin{tabular}{|c|c|c|c|c|}
\hline \multirow{2}{*}{ Ítem } & \multicolumn{4}{|c|}{ Factor } \\
\hline & 1 & 2 & 3 & 4 \\
\hline 26. Suelo dejar para mañana lo que tenía que hacer hoy. & 0.575 & 0.022 & -0.027 & -0.069 \\
\hline $\begin{array}{l}\text { 2. Soy una persona que muestra iniciativa a la hora de } \\
\text { emprender un proyecto. }\end{array}$ & 0.511 & -0.02 & 0.03 & -0.109 \\
\hline 3. Estoy motivado con mi vida. & 0.51 & 0.079 & -0.027 & -0.13 \\
\hline $\begin{array}{l}\text { 31. Me gusta participar de actividades que impliquen } \\
\text { concentración. }\end{array}$ & 0.506 & 0.008 & -0.142 & 0.058 \\
\hline $\begin{array}{l}\text { 32. Me intereso por las actividades que impliquen mi } \\
\text { esfuerzo y creatividad. }\end{array}$ & 0.488 & 0.02 & -0.102 & 0.047 \\
\hline $\begin{array}{l}\text { 12. Ante un problema en mi vida, espero que se resuelva } \\
\text { por intervención de otros. }\end{array}$ & 0.482 & -0.08 & -0.005 & 0.138 \\
\hline 6. He perdido interés por el estudio, el trabajo. & 0.481 & 0.01 & -0.006 & -0.016 \\
\hline $\begin{array}{l}\text { 1. Me caracterizo por ser una persona que consigue las } \\
\text { cosas por esfuerzo propio. }\end{array}$ & 0.468 & -0.041 & -0.029 & -0.03 \\
\hline 22. Me esfuerzo poco en las cosas que hago. & 0.466 & 0.012 & 0.107 & -0.047 \\
\hline $\begin{array}{l}\text { 30. Yo lo que quiero, es hacer cosas que no impliquen } \\
\text { mucho esfuerzo. }\end{array}$ & 0.464 & -0.014 & -0.022 & 0.1 \\
\hline $\begin{array}{l}\text { 28. El futuro no me preocupa por ahora, ya veré luego que } \\
\text { hago. }\end{array}$ & 0.458 & -0.03 & 0 & -0.069 \\
\hline $\begin{array}{l}\text { 20. Las personas cercanas tienen la idea que soy una } \\
\text { persona cumplida. }\end{array}$ & 0.457 & -0.073 & 0.009 & 0.007 \\
\hline $\begin{array}{l}\text { 18. Haciendo las cosas me demoro mucho porque actúo } \\
\text { con lentitud a lo que debo hacer. }\end{array}$ & 0.457 & 0.044 & -0.086 & 0.158 \\
\hline 21. Tengo que obligarme para hacer algo. & 0.446 & -0.056 & 0.087 & 0.084 \\
\hline
\end{tabular}


Ítem
17. Pospongo las tareas que tengo.
23. Podría pasar el día sin hacer nada y sin sentir arrepentimiento.

11. No actúo cuando debería hacerlo.

29. Lo que requiera esfuerzo o mucha concentración no lo hago.

27. Pienso constantemente en el futuro.

5. Me interesa aprender cosas nuevas.

8. Tengo actividades en mi vida diaria que me animan.

4. Me cuesta trabajo iniciar algo nuevo.

9. No hago las cosas por mí mismo, dejo obrar a los demás.

24. Me cuesta levantarme en las mañanas y

frecuentemente me quedo en la cama más tiempo del planeado.

13. Estoy menos preocupado por mis problemas de lo que debería ser.

10. Me cuesta dirigir mi propia vida.

19. Constantemente he recibido críticas de personas cercanas por no terminar las tareas que me fueron asignadas.

53. Me emociono con las cosas que me suceden.

16. No siento inclinación, ni rechazo hacia las cosas.

15. Lo que me pasa me tiene sin cuidado.

25. Solo me interesa el aquí y el ahora.

14. Poco me interesa lo que le sucede a los demás.

55. Soy afectuoso con las personas significativas en mi vida.
Factor

$\begin{array}{cccc}\mathbf{1} & \mathbf{2} & \mathbf{3} & \mathbf{4} \\ 0.439 & -0.085 & 0.029 & 0.001\end{array}$

$\begin{array}{llll}0.437 & 0.013 & 0.007 & -0.149\end{array}$

$\begin{array}{llll}0.423 & 0.025 & 0.092 & -0.022\end{array}$

$\begin{array}{llll}0.405 & 0.095 & -0.075 & 0.165\end{array}$

$\begin{array}{llll}0.405 & 0.003 & -0.044 & -0.065\end{array}$

$\begin{array}{llll}0.404 & -0.032 & -0.151 & 0.056\end{array}$

$\begin{array}{llll}0.395 & 0.103 & -0.07 & -0.032\end{array}$

$\begin{array}{llll}0.388 & -0.021 & 0.067 & -0.071\end{array}$

$\begin{array}{llll}0.372 & -0.093 & 0.068 & 0.057\end{array}$

$\begin{array}{llll}0.357 & -0.058 & 0.033 & 0.024\end{array}$

$\begin{array}{llll}0.348 & -0.031 & 0.104 & -0.049\end{array}$

$\begin{array}{llll}0.345 & 0.035 & 0.14 & -0.039\end{array}$

$\begin{array}{llll}0.34 & -0.035 & 0.063 & 0.143\end{array}$

$\begin{array}{llll}0.325 & 0.055 & -0.01 & -0.012\end{array}$

$\begin{array}{llll}0.313 & 0.018 & 0.029 & 0.001\end{array}$

$\begin{array}{llll}0.307 & 0.087 & 0.046 & -0.055\end{array}$

$\begin{array}{llll}0.303 & -0.002 & -0.004 & -0.026\end{array}$

$\begin{array}{llll}0.293 & 0.08 & 0.054 & -0.101\end{array}$

$\begin{array}{llll}0.288 & 0.114 & 0.076 & 0.005\end{array}$ 


\begin{tabular}{|c|c|c|c|c|}
\hline \multirow{2}{*}{ Ítem } & \multicolumn{4}{|c|}{ Factor } \\
\hline & 1 & 2 & 3 & 4 \\
\hline $\begin{array}{l}\text { 7. Las personas cercanas me manifiestan que he perdido } \\
\text { interés en los asuntos cotidianos. }\end{array}$ & 0.217 & 0.074 & 0.171 & 0.024 \\
\hline $\begin{array}{l}\text { 46. Ahora me cuesta iniciar conversaciones, lo que no me } \\
\text { ocurría antes. }\end{array}$ & -0.043 & 0.863 & -0.041 & -0.097 \\
\hline 47. Antes sostenía una conversación, ahora es más difícil. & -0.028 & 0.725 & -0.048 & 0.095 \\
\hline $\begin{array}{l}\text { 45. Siento que antes era más expresivo y comunicativo } \\
\text { que ahora. }\end{array}$ & -0.086 & 0.69 & 0.017 & 0.002 \\
\hline 49. Me he vuelto más retraído socialmente. & -0.015 & 0.612 & 0.087 & 0.016 \\
\hline 52. En los últimos años he reducido mi círculo de amigos. & 0.03 & 0.458 & -0.09 & -0.01 \\
\hline 48. Se me dificulta expresar mis ideas. & 0.124 & 0.419 & 0.101 & 0.053 \\
\hline 51. Me relaciono solo con personas consumidoras. & 0.006 & 0.292 & 0.009 & -0.028 \\
\hline $\begin{array}{l}\text { 33. He notado que he venido descuidando mi aspecto } \\
\text { personal. }\end{array}$ & -0.056 & -0.021 & 0.823 & -0.014 \\
\hline $\begin{array}{l}\text { 34. He recibido críticas de personas cercanas o familiares, } \\
\text { porque he abandonado un poco mi aspecto personal. }\end{array}$ & -0.115 & -0.007 & 0.725 & 0.038 \\
\hline $\begin{array}{l}\text { 35. Me caracterizo por ser una persona que cuida de su } \\
\text { aspecto personal. }\end{array}$ & 0.089 & -0.056 & 0.597 & -0.038 \\
\hline 36. Poco me interesa el aspecto físico que tengo. & 0.027 & 0.04 & 0.409 & 0.016 \\
\hline 56. Me cuesta expresar emociones. & 0.156 & 0.2 & 0.232 & 0.022 \\
\hline $\begin{array}{l}\text { 38. La frecuencia de mis relaciones sexuales, han } \\
\text { disminuido debido a mi desinterés sexual. }\end{array}$ & 0.101 & 0.087 & 0.123 & 0.076 \\
\hline 44. Mis movimientos son más lentos ahora que antes. & -0.056 & 0.044 & 0.033 & 0.791 \\
\hline 43. Reacciono lentamente. & 0.024 & -0.023 & 0.015 & 0.738 \\
\hline 42. Noto que se me han disminuido mis reflejos. & -0.039 & 0.005 & 0.03 & 0.72 \\
\hline 41. Camino lentamente y sin prisa. & -0.118 & -0.036 & -0.073 & 0.201 \\
\hline
\end{tabular}


Los ítems resultantes en el análisis factorial con carga superior a .5 fueron sometidos a análisis de fiabilidad, obteniendo un alpha de Cronbach de .82. Con estas preguntas, en cuatro factores se obtiene un $58.8 \%$ de la varianza explicada.

\section{Discusión}

Los resultados indican que existe una variable latente relacionada con la disminución de la motivación en consumidores de marihuana, aislando estos efectos de otras condiciones que podrían tener efecto sobre la motivación como el síndrome prefrontal, la depresión, el aplanamiento afectivo por un trastorno mental y el consumo perjudicial de alcohol. La existencia de la afectación emocional de consumidores frecuentes de marihuana podría tener implicaciones para la provisión de tratamientos en el contexto de mayores incidencias de trastornos por consumo de sustancias en el mundo (Roussos, Gómez Penedo y Olivera Ryberg, 2017).

Aunque esto requiere de más investigación, tal deficiencia amotivacional no estaría determinada por la condición socioeconómica, el nivel educativo, el tipo de marihuana consumida ni su cantidad. Esto último es particularmente llamativo, puesto que sería de esperarse que a mayor consumo de marihuana, mayor deficiencia en la motivación. La solución al dilema podría considerar varias posibilidades: una es que la deficiencia en la motivación de consumidores de marihuana esté dada por otras causas, como factores de personalidad, que no fueron incluidas en este estudio. Otra posibilidad es que haya faltado precisión al momento de contestar la pregunta. Esto porque la presentación de la marihuana en formato cigarrillo corresponde a un estándar informal donde es difícil establecer la cantidad en gramos de marihuana, el del tráfico en la calle, por lo que el consumidor difícilmente podría estar seguro de cuánto producto consume. Lo segundo es que las fracciones de producto (por ejemplo, medio cigarrillo) son susceptibles de mayores errores de apreciación por parte del usuario.

Por lo anterior, sería importante calibrar mejor la pregunta acerca de la cantidad consumida, llevado a los participantes en un estudio de estas características a contestar por un periodo de tiempo más cercano (la última semana, por ejemplo), o presentar maquetas de cigarrillos como se venden en la calle, o los estándares armados por ellos, para una mejor representación.

Con respecto a la confiabilidad arrojada por la prueba, los valores obtenidos indican estabilidad de la medida. Así mismo, el índice de separación muestra que habría por lo menos tres grupos de personas separados por el conjunto de preguntas. Luego del tratamiento a las categorías de respuesta, la mayoría de los ítems mostraron buenos indicadores de ajustes, incluyendo un índice de discriminación cercando a uno, lo que se considera apropiado (Bond y Fox, 2015; Linacre, 2017).

El conjunto de preguntas sobre comportamientos y situaciones que pudieran revelar disminución en la motivación, una vez analizadas aplicando el modelo de Rasch, fueron llevadas a un análisis factorial que arrojó cuatro factores. El primero de ellos podría asimilarse al tipo de apatía y desinterés asociados con el síndrome amotivacional, que para este caso sería una apatía de tipo emocional, según la clasificación de Stuss, Van Reekum y Murphy (2000), la cual constituye una reducción de los comportamientos o actos dirigidos por objetivos propios, donde se produce falta de motivación, bien por reducción de la voluntad a ejecutar acciones, por la incapacidad de acabar las tareas o por la incapacidad de evaluar las consecuencias de los futuros actos, pero sin que estén afectadas las emociones (Robert et al., 2008). Al respecto, es importante recordar que en la fase final de análisis se excluyeron los sujetos que tenían aplanamiento afectivo, propio de trastornos mentales, evaluado mediante la Escala de Apatía de Marin (1991), la cual corresponde a una apatía conductual, según la clasificación de Stuss, Van Reekum y 
Murphy, corroborándose que el tipo de apatía relacionada con el consumo de marihuana sería de tipo emocional, lo que corresponde a la afectación de la zona orbito-medial de la corteza prefrontal (Eslinger y Damasio, 1985) y la zona límbica de los ganglios basales (estriado ventral y pálido ventral), es decir, el circuito límbico corticotálamo-estriatal, las mismas zonas donde actúa el THC (Moore et al., 2007).

El segundo factor estaría definido por disminución en la comunicación con retraimiento social, mientras el tercer factor haría referencia a descuido en la imagen personal. Finalmente, el cuarto factor estaría definido por una diminución en la rapidez de los movimientos. Todos estos signos han sido identificados en distintas revisiones, como parte del conjunto de afectaciones a la motivación producidos por el consumo consuetudinario de marihuana (Bobes y Calafat, 2000; Gutiérrez-Rojas, De Irala y Martínez-González, 2006; Karila et al., 2014; Rovai et al., 2013; Tziraki, 2012).

La baja en la motivación por el consumo de marihuana puede ser el resultado de los efectos que tiene el uso repetido de drogas en el circuito cerebral de recompensa, puesto que se ha descrito cómo la motivación hacia diversas áreas de la vida disminuye en la medida en que la motivación hacia la consecución y el consumo de sustancias aumenta (Arias-Carrión, Stamelou, Murillo-Rodríguez, Menéndez-González y Pöppel, 2010; Calipari et al., 2017). Esto, a su vez, tiene implicaciones sobre la planeación de actividades y la previsión de recompensas futuras por actividades complejas que impliquen organización y esfuerzo, lo que estaría relacionado con las conexiones del circuito de recompensa con la corteza prefrontal (Goldstein y Volkow, 2002).

A pesar de lo anteriormente indicado, la evidencia aquí presentada no es concluyente. El mapa de personas e ítems muestra que hay un desfase entre dos coordenadas claves en el modelo de Rasch: la dificultad de las preguntas y la habilidad de las personas. En otras pala- bras, las preguntas parecían dirigirse a niveles de desmotivación muy altos en comparación con el grado de desmotivación de las personas. Esto indica que tal vez sería necesario revisar las preguntas formuladas y añadir algunas que representen mejor el constructo. En todo caso, las preguntas formuladas logran medir la deficiencia en motivación en aproximadamente la mitad del grupo estudiado.

El desfase entre ítems y personas también puede ser el resultado de otro asunto: la gran cantidad de ítems en los cuales la escala va de un sentido a otro. Aunque es de usanza común el presentar ítems que por su redacción implican calificar con la escala de la prueba invertida, diversos autores han mostrado que esto produce sesgos en las respuestas (Conrad et al., 2004; Weijters, Baumgartner y Schillewaert, 2013).

Por otra parte, el uso de categorías de respuesta del tipo nunca, pocas veces, a veces sí a veces no y siempre, mostró no ser lo más adecuado Por ejemplo, la distancia entre pocas veces y a veces sí y a veces no, es muy subjetiva y podría producir respuestas en un valor o en otro, indistintamente. La aplicación del modelo de Rasch permite identificar este problema y darle un tratamiento. Sin embargo, la transformación de las categorías de respuesta hace perder la intención original de contar con un conjunto de valores similar al de una escala del tipo Likert.

Igualmente, aunque el conjunto de preguntas fue planteado después de una revisión de la literatura y de la consulta a expertos, se observa que podría ser necesario construir nuevos ítems que midan mejor el grado de motivación de los individuos. Para esto, sería útil recurrir a los mismos consumidores de marihuana, tomando su propia descripción acerca de posibles deficiencias en la motivación, para así mejorar la validez de los ítems y lograr un mejor recorrido por el constructo que se quiere medir (De Vellis, 2017; Henao y Pérez, 2013).

Para terminar, es necesario anotar que este trabajo fue útil para indicar comportamientos 
y situaciones que indican la deficiencia en motivación entre consumidores de marihuana, debido al recurso de una muestra de tamaño considerable de usuarios de la sustancia, con la introducción de instrumentos que permitieron excluir a los participantes que presentaron otros eventos como depresión, síndrome prefrontal, apatía provocada por otros trastornos mentales y alcoholismo.

En todo caso, no se resuelve la controversia acerca de la existencia del síndrome amotivacional en consumidores de marihuana. Para profundizar en estos asuntos, podrían desarrollarse otros estudios en los que se incluyeran sujetos no consumidores de marihuana $\mathrm{y}$ sujetos consumidores donde se tuvieran en cuenta además otros aspectos como la frecuencia y la cantidad de consumo (consumo pesado o liviano), la edad de inicio en el consumo, niveles de $\Delta$ 9-tetrahidrocannabinol y variables ambientales como los contextos socioculturales de los consumidores, que de por sí pueden producir falta de motivación. Así mismo, usar una prueba alterna de motivación y el criterio clínico para dar un paso adicional hacia un instrumento que permita medir con mayor certeza el síndrome amotivacional por consumo de marihuana.

\section{Referencias bibliográficas}

American Psychiatric Association (Ed.). (2013). Diagnostic and statistical manual of mental disorders: DSM-5 (5th ed). Washington, D.C: American Psychiatric Association.

Arias-Carrión, O., Stamelou, M., Murillo-Rodríguez, E., Menéndez-González, M. y Pöppel, E. (2010). Dopaminergic reward system: a short integrative review. International Archives of Medicine, 3(1), 24. http://dx.doi. org/10.1186/1755-7682-3-24

Babor, T., Higgins-Biddle, J., Saunders, J. y Monteiro, M. (2001). AUDIT. The Alcohol Use Disorders Identification Test. Guidelines for use in primary care (2nd ed). Geneva: World Health Organization.

Barnwell, S.S., Earleywine, M. y Wilcox, R.
(2006). Cannabis, motivation, and life satisfaction in an internet sample. Substance Abuse Treatment, Prevention, and Policy, 1, 2. http:// dx.doi.org/10.1186/1747-597X-1-2

Beck, A.T., Steer, R.A., Brown, G.K., Sanz, J. y Vázquez, C. (2011). BDI-II: Inventario de Depresión de Beck-II. Madrid: Pearson Educación.

Bobes, J. y Calafat, A. (2000). De la neurolobiología a la psicosociología del uso-abuso del cannabis. Adicciones, 12(5), 7. http://dx.doi. org/10.20882/adicciones.669

Bond, T.G. y Fox, C.M. (2015). Applying the Rasch model: fundamental measurement in the human sciences (3rd ed.). New York: London: Routledge, Taylor and Francis Group.

Calipari, E.S., Juarez, B., Morel, C., Walker, D.M., Cahill, M.E., Ribeiro, E., ... Nestler, E.J. (2017). Dopaminergic dynamics underlying sex-specific cocaine reward. Nature Communications, 8, 13877. http://dx.doi.org/10.1038/ ncomms 13877

Conrad, K.J., Wright, B.D., McKnight, P., McFall, M., Fontana, A. y Rosenheck, R. (2004). Comparing traditional and Rasch analyses of the Mississippi PTSD Scale: Revealing limitations of reverse-scored items. Journal of Applied Measurement, 5(1), 15-30.

DeVellis, R.F. (2017). Scale development: theory and applications (Fourth edition). Los Angeles: SAGE.

Eslinger, P. y Damasio, A. (1985). Severe disturbance of higher cognition after bilateral frontal lobe ablation: patient EVR. Neurology, 35(12), 1731-1741.

García Carretero, M.Á., Novalbos Ruiz, J.P., Martínez Delgado, J.M. y O'Ferrall González, C. (2016). Validación del test para la identificación de trastornos por uso de alcohol en población universitaria: AUDIT y AUDIT-C. Adicciones, 28(4), 194. http://dx.doi.org/10.20882/ adicciones. 775

Gobierno de Colombia. (2018). Estudio nacional de consumo de sustancias psicoactivas en población escolar. Colombia 2016. Bogotá DC.: Observatorio de Drogas de Colombia.

Gold, M. (1991). Marihuana. Barcelona: 
Ediciones en Neurociencias.

Goldstein, R.Z. y Volkow, N.D. (2002). Drug Addiction and Its Underlying Neurobiological Basis: Neuroimaging Evidence for the Involvement of the Frontal Cortex. American Journal of Psychiatry, 159(10), 1642-1652. http://dx.doi.org/10.1176/appi.ajp.159.10.1642

Gutiérrez-Rojas, L., De Irala, L. y Martínez-González, M. (2006). Efectos del cannabis sobre la salud mental en jóvenes consumidores. Revista de Medicina Universidad de Navarra, 50(1), 3-10.

Hall, W., Solowij, N. y Lemon, J. (1994). The health and psychological effects of cannabis use. National Drug Strategy Monograph Series No. 25. Australian Government Publication Service.

Henao, C. y Pérez, J. (2013). Validez de apariencia y concurrente de un instrumento de evaluación de la discapacidad en personas con lesión medular crónica, basado en el core set abreviado de la CIF. Revista Ciencias de la Salud, 11, 147-161.

Karila, L., Roux, P., Rolland, B., Benyamina, A., Reynaud, M., Aubin, H.J. y Lançon, C. (2014). Acute and long-term effects of cannabis use: a review. Current Pharmaceutical Design, 20(25), 4112-4118.

Lac, A. y Luk, J.W. (2017). Testing the Amotivational Syndrome: marijuana use longitudinally predicts lower self-efficacy even after controlling for demographics, personality, and alcohol and cigarette. Prevention Science, 19(2) 117-126. http://dx.doi.org/10.1007/ s11121-017-0811-3

Lawn, W., Freeman, T.P., Pope, R.A., Joye, A., Harvey, L., Hindocha, C., ... Curran, H.V. (2016). Acute and chronic effects of cannabinoids on effort-related decision-making and reward learning: an evaluation of the cannabis 'amotivational' hypotheses. Psychopharmacology, 233(19-20), 3537-3552. http://dx.doi. org/10.1007/s00213-016-4383-x

Linacre, J.M. (2017). WINSTEPS Rasch measurement computer program. Chicago: Winsteps.com.

Lloret-Segura, S., Ferreres-Traver, A., Hernández-Baeza, A. y Tomás-Marco, I. (2014). El análisis factorial exploratorio de los ítems: una guía práctica, revisada y actualizada. Anales de Psicología, 30(3), 1151-1169. http://dx.doi. org/10.6018/analesps.30.3.199361

Marin, R.S. (1991). Apathy: a neuropsychiatric syndrome. The Journal of Neuropsychiatry and Clinical Neurosciences, 3(3), 243-254. http://dx.doi.org/10.1176/jnp.3.3.243

Marin, R.S., Biedrzycki, R.C. y Firinciogullari, S. (1991). Reliability and validity of the Apathy Evaluation Scale. Psychiatry Research, 38(2), 143-162.

McGlothlin, W. y West, L. (1968). The marijuana problem: an overview. American Journal of Psychiatry, 282, 24-34.

Medina, A.L., Arévalo, N.M., Beltrán, S.D., Chavarro, Y.L., Herazo, E. y Campo-Arias, A. (2014). Consistencia interna y estructura interna del cuestionario AUDIT en amerindios. Revista Investigaciones Andina, 16(28), 871-880.

Moore, T.H., Zammit, S., Lingford-Hughes, A., Barnes, T.R., Jones, P.B., Burke, M. y Lewis, G. (2007). Cannabis use and risk of psychotic or affective mental health outcomes: a systematic review. The Lancet, 370(9584), 319-328. http://dx.doi.org/10.1016/S0140$\underline{6736(07) 61162-3}$

Morales Vallejo, P. (2013). El Análisis Factorial en la construcción e interpretación de tests, escalas y cuestionarios. Madrid: Universidad Pontificia Comillas.

Robert, P.H., Berr, C., Volteau, M., Bertogliati-Fileau, C., Benoit, M., Guerin, O., ... PréAL Study Group. (2008). Importance of lack of interest in patients with mild cognitive impairment. The American Journal of Geriatric Psychiatry: Official Journal of the American Association for Geriatric Psychiatry, 16(9), 770-776. $\quad$ http://dx.doi.org/10.1097/JGP. $\underline{0 \mathrm{~b} 013 \mathrm{e} 31817 \mathrm{e} 73 \mathrm{db}}$

Roussos, A.J., Gómez Penedo, J.M. y Olivera Ryberg, J. (2017). Satisfacción de los pacientes en tratamientos por abuso de sustancias y su relación con variables de las instituciones asistenciales. Interdisciplinaria, 34(1), 193-210. http://dx.doi.org/10.16888/interd.2017.34.1.12 
Rovai, L., Maremmani, A., Pacini, M., Pani, P.P., Rugani, F., Lamanna, F., ... Maremmani, I. (2013). Negative dimension in psychiatry. Amotivational syndrome as a paradigm of negative symptoms in substance abuse. Rivista di Psichiatria, 48(1), 1-9. http://dx.doi. org/10.1708/1228.13610

Ruiz-Sánchez de León, J.M., Pedrero-Pérez, E.J., Lozoya-Delgado, P., Llanero-Luque, M., RojoMota, G. y Puerta-García, C. (2012). Prefrontal Symptoms Inventory for clinical evaluation of addictions in everyday life: development process and psychometric properties. Revista de Neurologia, 54(11), 649-663.

Scoppetta, O. (2010). Consumo de drogas en Colombia: características y tendencias. Bogotá DC.: Dirección Nacional de Estupefacientes.

Smith, D. (1968). Acute and chronic toxicity of marijuana. Journal of Psychodelic Drug, 2, 37-47.

Solé Puig, J. (2001). Desarrollo de la CIE-10 y el DSM-IV para trastornos por Cánnabis, Salud y Drogas 1(1), 41-70.

Stuss, D., Van Reekum, R. y Murphy, K. (2000). Differentiation of states and causes of apathy. En J. Borod (Ed.), The Neuropsychology of emotion (pp. 340-363). Oxford: Oxford University Press.

Tziraki, S. (2012). Trastornos mentales y afectación neuropsicológica relacionados con el uso crónico de cannabis. Revista de Neurología, 54(12), 750-760.
Valdés, C., Morales-Reyes, I., Pérez, J.C., Medellín, A., Rojas, G. y Krause, M. (2017). Propiedades psicométricas del inventario de depresión de Beck IA para la población chilena. Revista Médica de Chile, 145(8), 1005-1012. http://dx.doi.org/10.4067/s0034$\underline{98872017000801005}$

van Hell, H.H., Vink, M., Ossewaarde, L., Jager, G., Kahn, R.S. y Ramsey, N.F. (2011). Efectos crónicos del consumo de cannabis sobre el sistema de recompensa humano: un estudio de RMf. Psiquiatría Biológica, 18(2), 45-54. http://dx.doi.org/10.1016/j.psiq.2011.08.003

Volkow, N.D., Wang, G.J., Telang, F., Fowler, J.S., Alexoff, D., Logan, J., ... Tomasi, D. (2014). Decreased dopamine brain reactivity in marijuana abusers is associated with negative emotionality and addiction severity. Proceedings of the National Academy of Sciences, 111(30), E3149-E3156. http://dx.doi. org/10.1073/pnas.1411228111

Volkow, N.D., Swanson, J.M., Evins, A.E., DeLisi, L.E., Meier, M.H., Gonzalez, R., ... Baler, R. (2016). Effects of Cannabis Use on Human Behavior, Including Cognition, Motivation, and Psychosis: A Review. JAMA Psychiatry, 73(3), 292. http://dx.doi.org/10.1001/jamapsychiatry.2015.3278

Weijters, B., Baumgartner, H. y Schillewaert, N. (2013). Reversed item bias: An integrative model. Psychological Methods, 18(3), 320-334. http://dx.doi.org/10.1037/a0032121

Recibido: 30 de enero de 2018 Aceptado: 16 de diciembre de 2019 
